\title{
Investigación de Rasgos Dentales No Métricos en Poblaciones Sudamericanas Actuales: Estado de Situación y Contextualización Forense
}

\author{
Research on Non-Metric Dental Traits in Present South-American Populations: \\ State of Art and Forensic Contextualization
}

\author{
Sandra López-Lázaro*; Carolina Soto-Álvarez*; Guillermo Aramburú***; \\ Ivana Rodríguez"*; Mario Cantín",*** \& Gabriel M. Fonseca*,**
}

LÓPEZ-LÁZARO, S.; SOTO-ÁLVAREZ, C.; ARAMBURÚ, G.; RODRÍGUEZ, I.; CANTÍN, M. \& FONSECA, G. M. Investigación de rasgos dentales no métricos en poblaciones sudamericanas actuales: estado de situación y contextualización forense. Int. J. Morphol., 34(2):580-592, 2016.

RESUMEN: En las poblaciones humanas recientes existe una patrón de variación geográfico asociado a la morfología del diente. En las últimas décadas se ha generado una notable producción científica en torno al estudio de los rasgos dentales no métricos con el fin de definir los patrones geográficos de los grupos, su historia, origen o estructura, entre otros. Entre las aplicaciones propuestas está la posibilidad del uso de la frecuencia de los diferentes rasgos de expresión en un contexto forense. Se presenta una revisión de la literatura en las bases MEDLINE, SciELO, REDALYC y LILACS. Se incluyeron artículos completos, en español, portugués e inglés, que realicen análisis de rasgos morfológicos dentales en poblaciones sudamericanas actuales (siglos XX y XXI). Los artículos seleccionados fueron evaluados por dos investigadores de manera independiente. Las estrategias de búsqueda permitieron pesquisar 36 estudios con una importante participación de Colombia (tanto en autores como en muestreos), seguida por Chile y Brasil. Si bien estos resultados demuestran una robusta actividad en el ámbito de la antropología dentaria Sudamericana estudiando poblaciones actuales, se sugiere una reevaluación de las metodologías empleadas si se pretende asignar un valor forense a los rasgos dentales morfológicos.

PALABRAS CLAVE: Antropología dental; Rasgos no métricos; Sudamérica; Odontología forense.

\section{INTRODUCCIÓN}

Los dientes poseen características especiales que los convierten en un registro idóneo para fines identificatorios: (a) individualidad y variabilidad; (b) resistencia y durabilidad a agentes químicos, físicos, biológicos, mecánicos y térmicos; y (c) accesibilidad para su análisis. Estas particularidades otorgan un gran valor a los dientes con respecto a otras estructuras anatómicas: permiten el registro en poblaciones vivas, ya sea mediante la observación directa o a través de reproducciones en impresiones, o en poblaciones históricas gracias a la preservación sostenida en la dureza de los tejidos dentales.

Los rasgos morfológicos dentales coronarios son formas fenotípicas producto de procesos indirectos de se- creción mineral durante la odontogénesis, de carácter ontogénico, expresados y regulados por el genoma tanto de cada individuo como de una población determinada (Aguirre et al. 2007a, 2007b). Estos rasgos pueden representar estructuras positivas (tuberculares y radiculares) o negativas (intertuberculares y fosomorfas) con el potencial de estar o no presentes en una posición dental específica o manifestarse de diferente manera en uno o más miembros de un grupo específico de una población humana (Aguirre et al. 2007a, 2007b).

La primera vez que un carácter morfológico fue analizado mediante dicotomía de presencia/ausencia, tiene lugar en el trabajo sobre incisivos en pala que el antropólogo

" Laboratorio de Pericias en Odontología Forense, Centro de Investigación en Morfología Aplicada, Facultad de Odontología, Universidad de La Frontera, Temuco, Chile.

** Facultad de Odontología, Universidad Nacional de Córdoba. Córdoba, Argentina.

*** Centro de Investigación en Ciencias Biomédicas, Universidad Autónoma de Chile, Temuco, Chile.

Trabajo subsidiado por la Secretaría de Ciencia y Tecnología de la Universidad Nacional de Córdoba (S.E.C.y T.), Código de Incentivos 05/J146, resolución Nro. 203/14, Argentina.

Dirección de Investigación, Universidad de La Frontera, Proyecto DIE15-0007. 
checo, Ales Hrdlicka, publicado en 1920 (Scott \& Turner, 2000). Dahlberg en los '40 y Hanihara en los '60, estudiando denticiones permanentes y temporarias respectivamente mediante placas de impresión de diferentes rasgos, sentaron las bases del reconocido sistema ASUDAS (Arizona State University Dental Anthropology System) (Scott \& Turner). Estos estándares se basan en la creación de placas que representan físicamente estos diferentes atributos desde el mínimo al máximo grado de expresión. Para cada uno de los rasgos no métricos se facilita el nombre, el diente en que puede ser observado y un comentario con la definición o localización, entre otros (Turner et al. 1991). Son numerosas las investigaciones dedicadas al estudio de estos rasgos dentales no métricos (más de 100 descritos hasta el momento en corona y raíz) con el objeto de definir los patrones geográficos de los grupos, su historia, origen o estructura, entre otros (Rodríguez-Flórez, 2003). Los antropólogos dentales utilizan muchas de estas características morfológicas a través de estadísticas relativamente complejas para describir el grado de variabilidad que existe intra e interpoblacionalmente, a modo de conocer sus correspondencias, en especial las ancestro-descendientes (Edgar, 2013).

Al estudiar el desarrollo de la investigación sobre variación morfológica dental de poblaciones históricas sudamericanas, Fonseca et al. (2016) pusieron en evidencia que aunque el estudio de esos rasgos no métricos permite observar relaciones con la ancestría justificando su importancia en la identificación humana, existe un desequilibrio en la investigación de los diferentes países que componen el macizo sudamericano. Debido a la intensa popularización de la antropología biológica, la situación ética y jurídica de movimientos de comunidades nativas y el descubrimiento de fosas comunes de desaparecidos en contextos de vulneración de derechos humanos, los mismos autores sugieren revisar los estudios de ancestría y morfología en escenarios diferentes que las contextualicen con evolución, historia y fundamentalmente miscegenación si se pretende asignarles un valor forense. Esta hipotética asociación necesariamente debe ser realizada con estudios en poblaciones actuales (s. XX y XXI) si se pretende utilizar la frecuencia de los diferentes rasgos de expresión interpoblacionalmente, con el fin de determinar un ancestro común en un contexto forense (Alsoleihat, 2013). A pesar de que los antropólogos forenses están familiarizados con las limitaciones que ofrecen los rasgos morfológicos, y de que la actual utilidad de los mismos no ha sido formalmente puesta a prueba, un grupo de "aventureros" ha decido explorar el valor de estos rasgos poco conocidos (Edgar, 2009). Son diversos los investigadores que han tratado de buscar las herramientas estadísticas que permitan estimar el grupo poblacional de un individuo no identificado en un contexto judicial (Alsoleihat; Edgar, 2005, 2009, 2013; Edgar \& Ousley, 2010; Tinoco et al. 2016).
Se presenta una revisión respecto del desarrollo de la investigación sobre variación morfológica de poblaciones sudamericanas actuales utilizando rasgos dentales no métricos, y se discute su aplicabilidad para la estimación de ancestría y grupo poblacional en contextos forenses.

\section{MATERIAL Y MÉTODO}

Se realizó una revisión sistemática de la literatura en las bases de datos MEDLINE, SciELO, REDALYC y LILACS por dos investigadores de manera independiente, utilizando la siguiente combinación de términos ("teeth" OR “dental") AND "anthropology" AND ("morphology" OR "phylogeny" OR "traits") en español e inglés con respectivas estrategias de búsqueda. Éstas se realizaron entre el 15 y 25 de marzo de 2016 limitándose a estudios en inglés, español y portugués, donde se realizó el análisis de rasgos morfológicos externos dentales no métricos en poblaciones humanas actuales de Sudamérica en un contexto histórico datado en el siglo XX y XXI, esto para dar relación eventual forense entendiendo que momentos históricos previos deben ser considerados arqueológicos (Kovacevic \& Gruengold, 2010). Dentro de las categorías de estudio se consideraron el año y perfil de la publicación, la nacionalidad y caracterización profesional de los autores, países muestreados, tamaño muestral, instrumento para el análisis morfológico, cantidad de rasgos dentales empleados y sus resultados. Fueron excluidos los estudios que no especificaran los rasgos morfológicos analizados o aquellos que citaran estudios previos si se contaba con el estudio primario correspondiente. Los artículos seleccionados fueron evaluados en su calidad por dos investigadores de manera independiente siguiendo la guía de evaluación critica de la JAMA, resolviéndose por consenso aquellos casos donde existieron discrepancias.

\section{RESULTADOS}

Se encontraron 1970 artículos como resultado de la búsqueda, de los cuales 16 cumplieron la totalidad de los criterios de inclusión y presentaron la calidad metodológica necesaria según los estándares requeridos. Una búsqueda manual posterior permitió incluir otras 20 publicaciones que reunían similares criterios. En total se obtuvo un total de 36 artículos (Fig. 1).

Año y perfil de publicación (Tabla I). Se observaron dos fases netas cronológicas de presencia de publicaciones, la primera entre 1966 y 1981, y la segunda entre 2001 y 2016, separadas por un periodo de absoluta ausencia de tales re- 


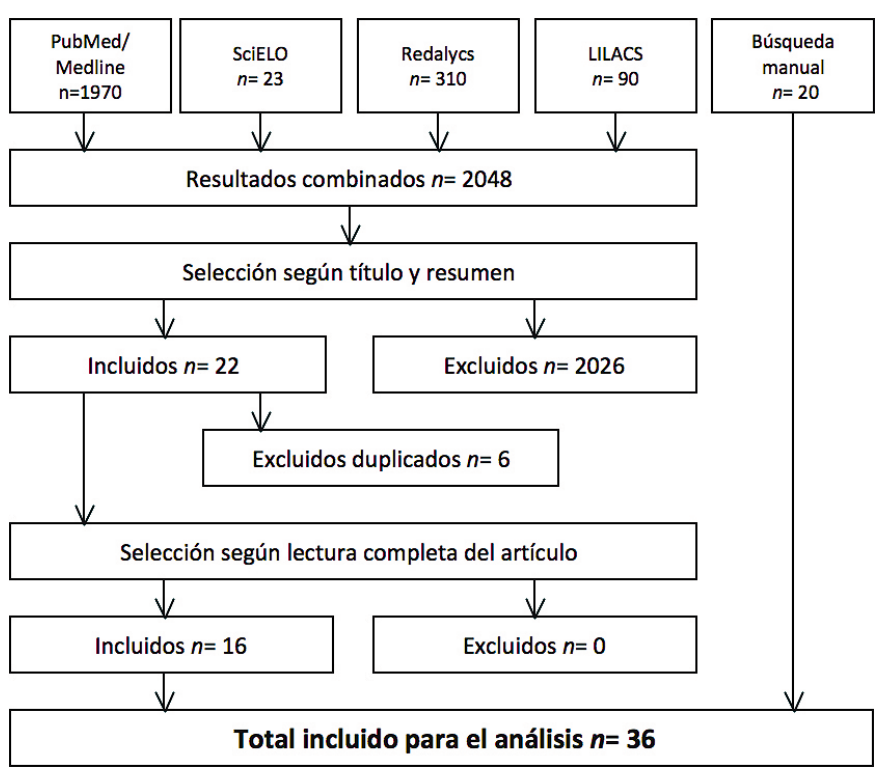

Fig. 1. Flujograma de la revisión de literatura y criterios para selección y exclusión de artículos.

gistros. Los años 2006 y 2007 ofrecieron el máximo de registros con 4 publicaciones cada año. Revista Estomatología (Colombia), Dental Anthropology Journal (Estados Unidos) y American Journal of Physical Anthropology (Estados Unidos) fueron las revistas con más publicaciones (4 cada una), seguidas por International Journal of Morphology (Chile) y Colombia Médica (Colombia) con 3 cada una, y HOMO Journal of Comparative Human Biology (Alemania), Journal of Dental Research (Estados Unidos), Human Biology (Estados Unidos), Revista de la Facultad de Odontología de la Universidad de Antioquía (Colombia) y Universitas Odontologica (Colombia) con 2 cada una.

Del total de 18 revistas identificadas, 6 fueron de Colombia, 4 de Estados Unidos, 2 de Alemania y 1 de Argentina, Canadá, Chile, Irlanda, México y Uruguay, respectivamente. En lo que refiere exclusivamente al perfil editorial de las revistas, 7 están dedicadas a la odontología general, 3 a la antropología general, 2 a la biología, 2 a la antropología dentaria, 2 a las ciencias forenses (una de ellas a la odontología forense), 1 a la morfología y 1 a la medicina.

Relacionando esta información con los periodos cronológicos mencionados, en la primera fase (1966-1981), las revistas de Estados Unidos lideraron con 3 publicaciones, seguidas por Alemania y Canadá con 1 respectivamente, con la antropología como perfil más frecuente seguida por biología y odontología con 3, 1 y 1 publicaciones respectivamente. En la segunda fase (2001-2016), el liderazgo absoluto lo tuvo Colombia con sus 6 revistas, seguida por Alemania, Argentina,
Chile, Estados Unidos, Irlanda, México y Uruguay con 1 cada una de ellas. En esta fase, odontología fue el perfil más frecuente con 6 revistas, seguida por antropología dental con 2, ciencias forenses con 2 (una de ellas dedicada a la odontología forense), y 1 para biología, medicina y morfología respectivamente.

Países y perfiles de los autores (Tabla I). Se identificaron un total de 111 autores destacando Colombia con 62, Chile con 15 (1 también con filiación de España), Brasil con 10, Argentina con 6, Estados Unidos con 5, Uruguay con 4, Paraguay con 3, Canadá y Sudáfrica con 2 e India (con filiación también de Estados Unidos) y Venezuela con 1. Se han observado 6 colaboraciones internacionales: 2 de Chile-India (con filiación Estados Unidos a través del genetista Dr. Ranajit Chakraborty, nacido en Bengala Occidental, actualmente académico de la Universidad de Cincinnati), Chile-Estados Unidos, Estados UnidosVenezuela, Argentina-Colombia y Brasil-Uruguay. Los autores con más publicaciones en la materia fueron el colombiano Freddy Moreno (quien firma 15 artículos), seguido por el chileno Francisco Rothhammer y la colombianas Luisa Aguirre, Diana Solarte y Diana Castillo con 3 artículos cada uno.

En lo que respecta a perfil de los autores, la mayoría fue impuesta por 93 odontólogos (57 de Colombia, 10 de Brasil, 8 de Chile, 6 de Argentina, 3 de Paraguay y Uruguay, 2 de Canadá y Sudáfrica y 1 de Venezuela, el prestigioso Dr. Charles Brewer-Carias, si bien dedicado durante 20 años a esa disciplina, luego reconocido como explorador y naturalista con importantes descubrimientos en el área de la biología). A estos perfiles siguieron 8 genetistas ( 7 de ellos con publicaciones entre 1968 y 1977, 5 de ellos de Chile), 3 antropólogos (todos de Colombia), 3 estadísticos, 2 fonoaudiólogos (Chile), 1 arqueólogo (Estados Unidos) y 1 epidemiólogo (Colombia).

\section{Países y tamaño de las muestras estudiadas (Tabla}

I, Fig. 2). La mayor cantidad de muestreos fueron realizados en Colombia, con un número de 20 artículos, seguido por Chile con 5, Brasil con 4 (dos de ellos sobre poblaciones Yanomami habitantes del norte brasileño y sur venezolano, únicos estudios identificados abarcando más de un país) (Brewer-Carias et al. 1976; Palomino et al. 1977), Argentina, Paraguay y Venezuela con 2 (este último país en referencia a los dos estudios mencionados) y Perú, Uruguay y Ecuador con 1. Excepción hecha de 4 reportes de casos aislados (Moreno \& Moreno, 2010; Rodríguez \& Moreno, 2006; Rodríguez-Flórez et al. 2006; Hernández et al. 


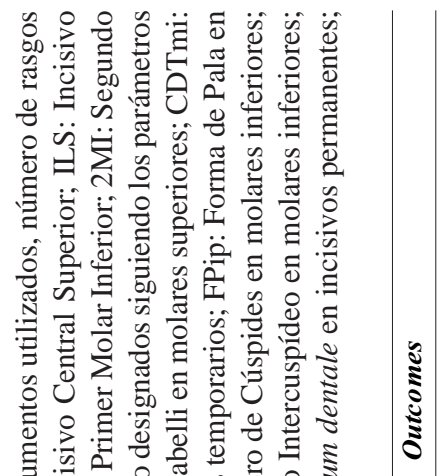

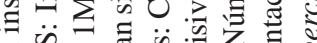

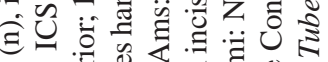

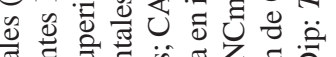

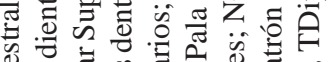

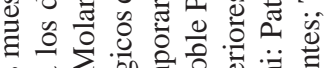

응

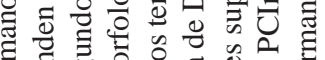

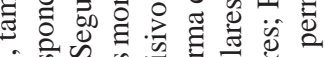

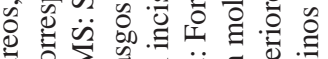

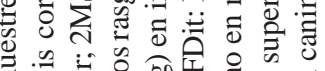

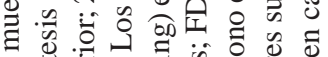

은

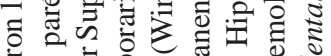

형

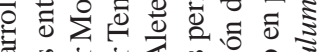

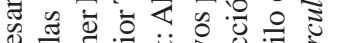

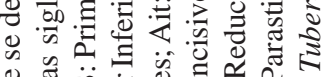

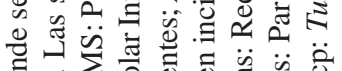

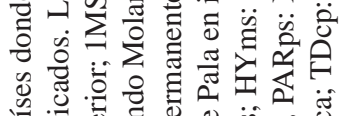

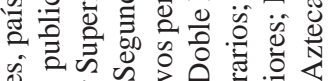

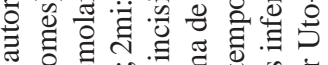

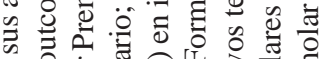

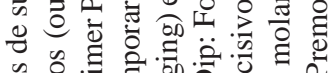

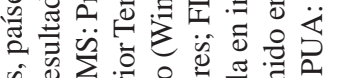

额

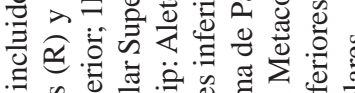

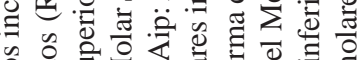

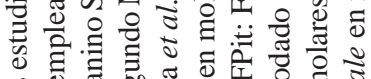

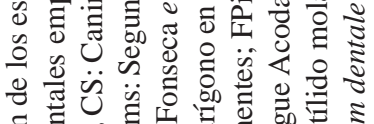

记

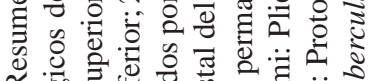

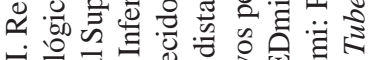

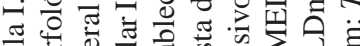

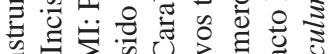

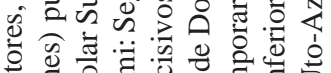

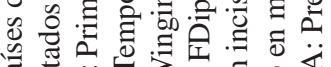

은
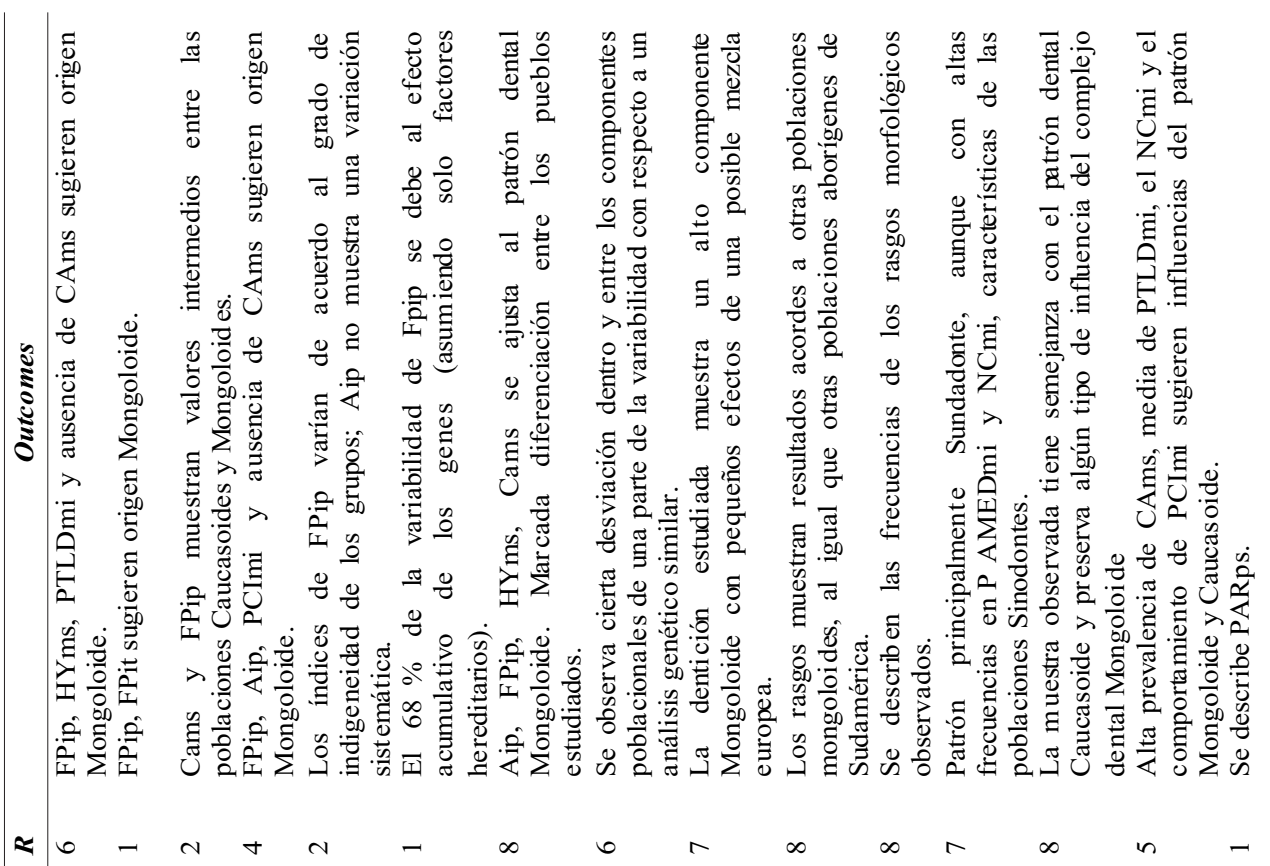

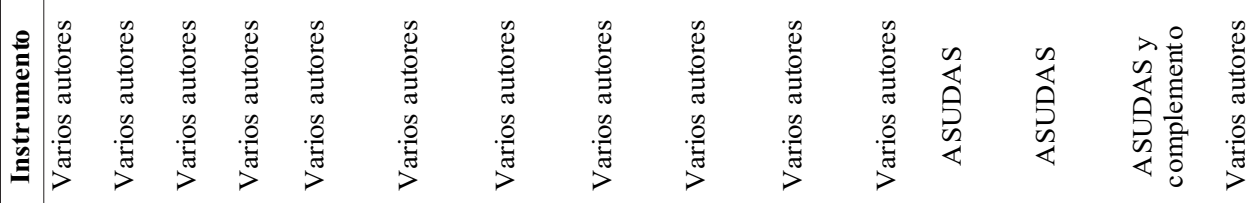

$=\hat{n}$ fै

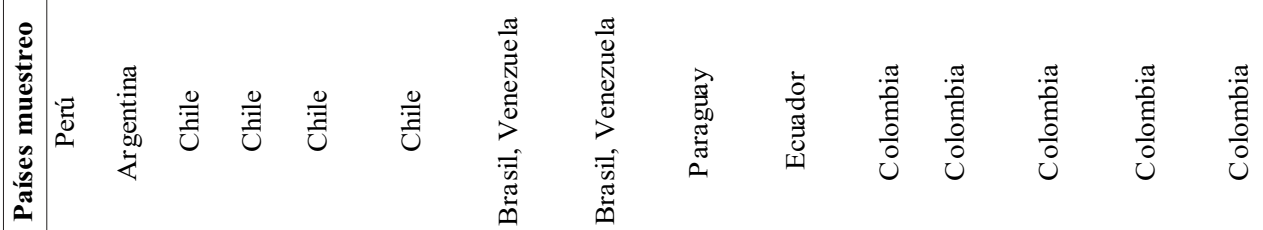

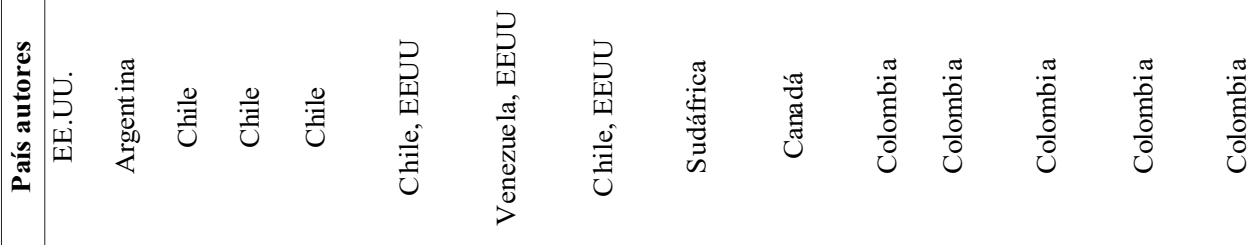

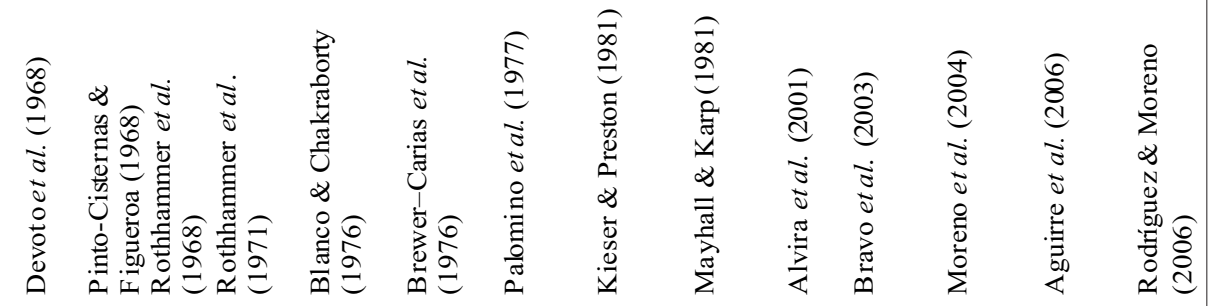

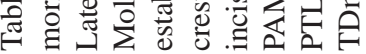




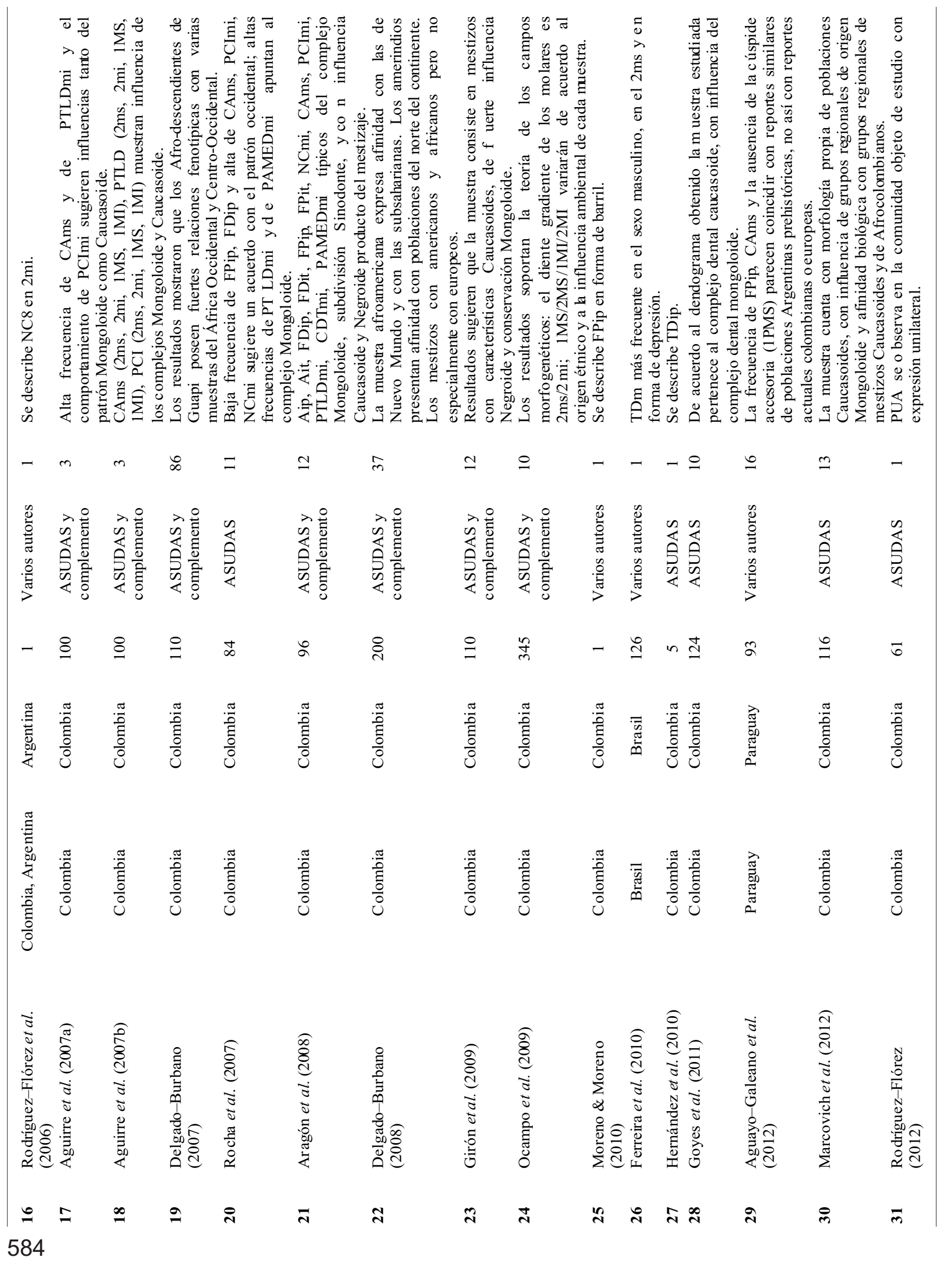



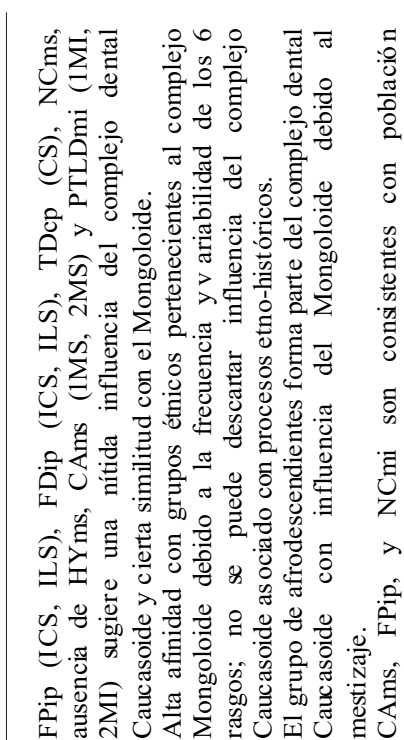

:을

욜. $\frac{\pi}{\sigma} \frac{\pi}{d} \cdot \frac{1}{4}$

\begin{tabular}{|c|c|c|c|}
\hline 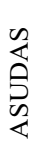 & 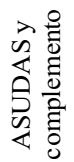 & $\underset{\infty}{\infty}$ & 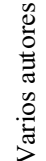 \\
\hline$n$ & ᄋ & O & $\stackrel{\infty}{\sim}$ \\
\hline
\end{tabular}

\begin{tabular}{|c|c|c|c|c|}
\hline 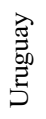 & $\frac{\frac{\pi}{0}}{\Xi}$ & $\frac{\pi}{0} \frac{\pi}{0}$ & $\frac{\stackrel{0}{\Xi}}{己}$ & $\begin{array}{l}\bar{\nabla} \\
\widetilde{\pi} \\
\bar{m}\end{array}$ \\
\hline
\end{tabular}

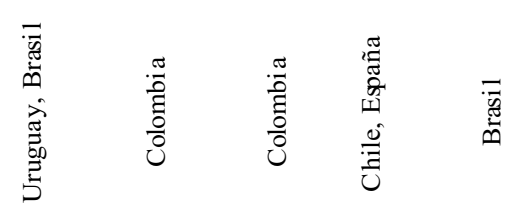

\begin{tabular}{|c|c|c|c|}
\hline 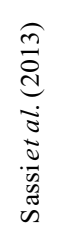 & 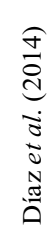 & 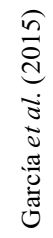 & 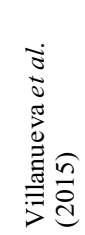 \\
\hline & $m$ & m & 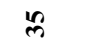 \\
\hline
\end{tabular}

2010), el tamaño muestral mínimo estuvo representado por 53 (Goaz \& Miller, 1966), y el máximo por 827 individuos (Pinto-Cisternas \& Figueroa, 1968) en Perú y Chile respectivamente.

En la primera fase cronológica (1966-1981) se observaron los mayores tamaños muestrales (esto incluyendo los 811 individuos Yanomami estudiados por Brewer-Carias et al. y Palomino et al. para Brasil-Venezuela), promediando un número de 427 individuos. En la segunda fase cronológica (2001-2016), sólo 2 artículos superaron los 300 individuos de muestra (Ocampo et al. 2009; Sassi et al. 2013) promediando un número de 117 individuos. Todos los reportes de caso se publicaron en esta segunda fase.

Instrumento de análisis (Tabla I). El estándar de ASUDAS fue utilizado en 19 artículos (en 10 como referencia única y en 9 complementado con otros reportes). Descartándolos 10 artículos publicados previamente a este estándar (Turner et al.), 7 artículos publicados entre 2001 y 2015 (Argentina, Brasil, Colombia -3-, Chile y Paraguay) no utilizaron o no informaron el empleo de ASUDAS.

Cantidad de rasgos analizados (Tabla I). Destaca Delgado-Burbano (2007), quien observó 86 y 37 rasgos morfológicos en sus trabajos de 2007 y 2008 respectivamente. Junto a estos dos estudios, AguayoGaleano et al. (2012), Aragón et al. (2008), Díaz et al. (2014), García et al. (2015), Girón et al. (2009), Ocampo et al. Marcovich et al. (2012) y Rocha et al. (2007) analizaron 10 o más rasgos. En el extremo opuesto con tan solo un rasgo no métrico estudiado -exceptuando los reportes de casos previamente señalados-, aparecen los trabajos de Devoto et al. (1968), Blanco \& Chakraborty (1976), Ferreira et al. (2010) y Rodríguez-Flórez (2012).

Outcomes (Tabla I). En la primera fase cronológica (1966-1981), los artículos se han centrado en el estudio de los rasgos morfológicos clásicos (forma de pala, doble pala, y ausencia de tubérculo de Carabelli), coincidiendo la mayoría en un origen Mongoloide de las muestras estudiadas. No obstante, Kieser \& Preston (1981) agregan a ese componente los pequeños efectos de una influencia europea y Pinto-Cisternas \& Figueroa muestran valores intermedios entre Caucasoides y Mongoloides.

Los artículos publicados durante la segunda fase cronológica (2001-2016) han destacado una mayor relación de las poblaciones sudamericanas con el complejo Caucasoide. Aguirre et al. (2006, 2007a, 2007b) señalan en sus trabajos la influencia tanto del patrón Mongoloide como del Caucasoide, y son diversos los estudios que sugieren una ubicación del complejo Caucasoide con influencia Mongoloide (García et al.; Goyes et al. 2011; Marcovich et al.; Moreno et al. 2004; Sassi et al.; Villanueva et al. 2015). De igual modo, Aragón et al. y Tinoco et al. establecen al complejo Mongoloide, pero además mostrando la influencia Caucasoide o europea junto a una influencia Negroide o de poblaciones africanas. Delgado-Burbano (2007, 2008), por su parte, establece relaciones entre poblaciones afrocolombianas y de la región subsahariana. 


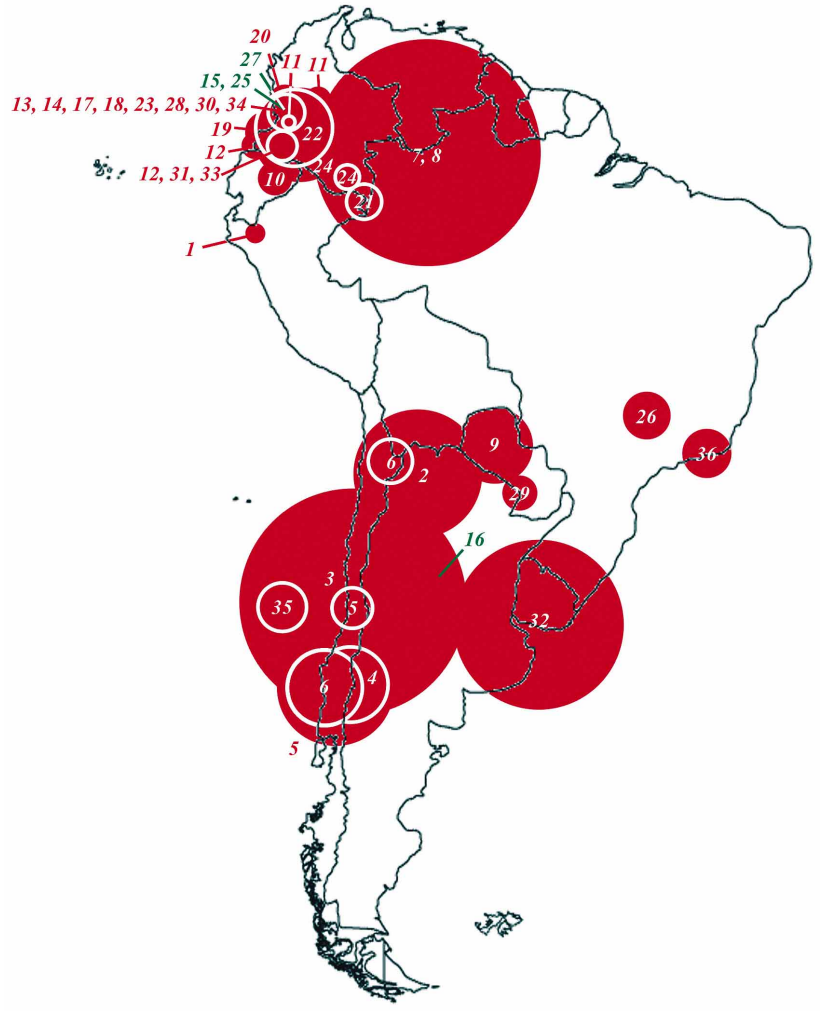

Fig. 2. Distribución geográfica de las poblaciones estudiadas por los artículos revisados (citas numéricas acordes a Tabla I). El tamaño de los círculos representa proporcionalmente el tamaño de las muestras empleadas. En verde reporte de casos.

\section{DISCUSIÓN}

Esta revisión pretende complementar los hallazgos reportados por Fonseca et al. cuando estudiaron el desarrollo de la investigación sobre variación morfológica de poblaciones sudamericanas utilizando rasgos dentales no métricos, esa vez en poblaciones históricas (previas al s. XX). La clara intención de esta nueva revisión es no sólo contrastar herramientas, interés o actitudes de la investigación focalizadas en una u otra población sudamericana (histórica versus actual), sino además establecer un estado de situación cuando los resultados de las mismas pretenden extrapolarse a contextos de aplicación forense. Más allá de los 36 artículos pesquisados, y aun reuniendo los criterios mínimos de calidad, 20 de ellos sólo pudieron ser detectados en búsquedas manuales direccionadas, algo probablemente motivado por la cobertura de las bases de datos, la indexación de las revistas o la definición de la pregunta de investigación, entre otros. Coincidimos con Fonseca et al. en la abundancia de literatura no convencional en la disciplina, experiencias no difundidas por canales tradicionales de publicación comercial o revistas no incluidas en bases de datos con la lógica inaccesibilidad, producción limitada y muchas veces no ajustada a normas de control bibliográfico, algo igualmente visible en esta revisión.

Nuevamente coincidiendo con lo reportado por Fonseca et al. han sido identificadas las mismas dos fases cronológicas de publicación de los estudios; una primera situada entre los años 1966-1981 y otra entre 2001 y 2016, más extensa y fructífera. Ambas se encuentran separadas por dos décadas de publicaciones ausentes (salvo una solitaria excepción publicada en 1995, recogida en la revisión mencionada). Pueden exponerse diferentes interpretaciones a este fenómeno; en la primera fase, la presencia de países desarrollados focalizando su atención en estas latitudes, sumada a las encomiables labores de investigadores argentinos y chilenos (los primeros dedicados a poblaciones históricas, los segundos a las actuales) ha permitido colocar presencia significativa en la comunidad científica internacional con referencias robustas e ineludibles. En el caso específico de Chile, la institucionalización de la antropología biológica chilena ocurrió en 1954 cuando se fundó el Centro de Estudios Antropológicos -posteriormente denominado Departamento de Antropología- en la Universidad de Chile en Santiago, entidad que contribuyó a desarrollar fuertemente la disciplina. Los aportes de Edmundo Covarrubias sobre flujo genético en poblaciones mapuches, William J. Schull (proveniente de la Universidad de Michigan, invitado por Covarrubias) quien ejerciera una importante influencia sobre genetistas que trabajaban en ese momento en la Universidad de Chile en Santiago y Valparaíso, entre ellos Juan Pinto-Cisternas, Carlos Campusano, Basilio Lazo, Hernán Palomino, Rafael Blanco y Francisco Rothhammer, junto a Schull, gestor del Programa Multinacional de Genética de Poblaciones Andinas desde 1972 hasta 1978, todos contribuyeron una abundante producción científica. Por su parte, Pinto-Cisternas, Blanco, Palomino y Campusano (estos últimos más interesados en antropología dental) suscribieron una intensa colaboración con biólogos humanos sudamericanos brasileños, argentinos y uruguayos que redundó en la fundación de la Asociación Latinoamericana de Antropología Biológica (ALAB), esfuerzo de integración multidisciplinaria con enfoques metodológicos y marcos conceptuales complementarios (Rothhammer \& Aspillaga, 2004).

Tomando en consideración la segunda fase (20012016), el número de publicaciones, las áreas geográficas de muestreo (Fig. 2), y más allá de la lógica revitalización que significaron las previas apariciones de la "Dental Anthropology Association" en 1986 (junto con la publicación de su "Dental Anthropology Journal") y la de ASUDAS en 1991 (Turner et al.), sin dudas el aporte de Colombia 
resulta significativo y particular de análisis. A diferencia de otros países latinoamericanos, la antropología dental colombiana inicia su trabajo recién en 1989 con la publicación del Cuaderno de Antropología No 19 de la Universidad Nacional titulado "Introducción a la Antropología Dental" por parte del antropólogo J. V. Rodríguez-Cuenca, obra que recoge toda la información disponible sobre variación morfológica y métrica dental humana (Moreno \& Moreno, 2007). Es a partir de allí y de la notable influencia del académico del Instituto de Etnología y Antropología de la Academia de Ciencias Rusa, Dr. A. Zoubov, luego de su conferencia "La antropología dental y la práctica forense" dictada en 1997 en el simposio "De lo prehispánico a lo forense: avances de la antropología biológica en Colombia", que la antropología colombiana puso epicentro en el estudio de la morbilidad oral en comunidades pre-hispánicas y en la investigación forense (Moreno \& Moreno, 2007). Para Rodríguez-Flórez (2005), es en torno al año 2000 que la divulgación de la antropología dental y sus aplicaciones en Colombia tomó fuerza a través del International Journal of Dental Anthropology y de la creación de diferentes grupos de investigación en varias universidades colombianas. Sin embargo, analizando los datos en la revisión que se presenta, no son precisamente los antropólogos colombianos quienes destacan por el número de publicaciones en esta segunda fase; de las 21 originadas por autores de ese país, sólo 5 son firmadas por antropólogos ( 3 de ellas con publicaciones en solitario) dejando 18 con participación de odontólogos, 15 de las cuales son encabezadas o conducidas por el Profesor de la Escuela de Odontología de la Universidad del Valle y de la Facultad de Ciencias de la Salud de la Pontificia Universidad Javeriana, ambas en Cali (Colombia), el Dr. Freddy Moreno. No es menor la mención del académico en este contexto, sin ninguna duda los 79 autores que firman esas 15 publicaciones y la notable presencia en ese listado de estudiantes del último año de diferentes facultades de odontología dan cuenta del firme impulso que el Dr. Moreno viene dando a la disciplina a través de las líneas de investigación bajo su dirección.

Es inevitable mencionar que la naturaleza de las muestras va a repercutir significativamente en el tamaño muestral disponible, con lo que contar con individuos vivos susceptibles de análisis va a resultar en el beneficio inestimable de muestras controlables y de mayor número si se las compara con las de poblaciones arqueológicas, dependientes éstas de la cantidad de restos hallados, su conservación, etc. Los datos revisados por Fonseca et al. y los observados en esta revisión parecen subrayar esta afirmación. Sin embargo, no es menor mencionar que para desarrollar investigaciones de orden clínico existen límites deontológicos, normas y leyes que explicitan cómo realizarlas y quiénes pueden por competencias. Moreno \& Moreno (2007) ase- veran que los rasgos morfológicos dentales, además de su valor antropológico, resultan de gran importancia clínica en el contexto odontológico, "ya que por su naturaleza pueden predisponer o favorecer el desarrollo de algún proceso patológico, en cuyo caso el plan de tratamiento debe partir de un correcto diagnóstico basado en el conocimiento del comportamiento del rasgo como factor etiológico". Coincidimos con los autores en que las herramientas que proporciona la antropología dental pueden resultar relevantes para odontólogos y especialistas que desean abordar una odontología basada en evidencia preventiva, diagnóstica y terapéutica, algo que parece corroborar la importante presencia de publicaciones en revistas de perfil odontológico. Sin embargo, y aunque Rodríguez-Flórez (2005) afirme que la antropología dental es una disciplina versátil en un amplio espectro de aplicación no sólo antropológica sino también odontológica (entre otras), los escasos 2 trabajos de colaboración entre odontólogos y antropólogos pesquisados en esta revisión (Moreno et al.; Rodríguez-Flórez et al.) demuestran que el (deseable) trabajo interdisciplinario como manera de resolver competencias potencialmente conflictivas si se trata de evaluar individuos en contextos clínicos, aún se mantiene en terreno de la utopía. Probable derivación de esto pueda significar la mención a las herramientas procedimentales empleadas en las investigaciones. Fonseca et al. defienden que, aunque ASUDAS y las reglas establecidas por Turner et al. logran globalizar contenidos y herramientas, su uso todavía sin adecuada difusión una vez que se traspasan los límites de la antropología. En esta revisión, exceptuando los 3 reportes inusuales de Moreno \& Moreno (2010), Rodríguez \& Moreno y Rodríguez-Flórez et al. (quienes buscan reformular las nóminas en relación a casos inusuales o no reportados previamente), en 4 publicaciones firmadas por odontólogos, ASUDAS no ha sido utilizada como marco metodológico lo que dificulta notablemente la posibilidad de contrastar esos resultados globalmente. Según indican Scott \& Turner, hasta el 2006 se habían distribuido 242 conjuntos de placas en 36 países (más de la mitad distribuidas en los Estados Unidos); no obstante, de Sudamérica solo Argentina, Chile y Brasil figuran como adquisidores de las mismas.

Los estudios de restos óseos y dentales de aborígenes americanos han mostrado una gran variabilidad en estas poblaciones, enmarcándolas en un patrón Mongoloide, aunque con algunas particularidades autralomelanésicos y caucasoides (Rodríguez-Cuenca, 2003). El "complejo dental Mongoloide", fue descrito por Hanihara en 1968 basándose en observaciones de cuatro rasgos dentales en japoneses, indios americanos y esquimales, y propuesto como característico del este asiático. Este complejo se subdivide, a su vez, en Sinodonte, propio del norte y con un patrón complejo y el Sundadonte, propio del sudeste asiático y con ras- 
gos más simplificados (Scott \& Turner). Los orígenes de la variabilidad americana se ha interpretado en torno a tres modelos evolutivos: (i) el modelo migracionista, plantea diferentes oleadas migratorias desde Asia; (ii) el modelo microevolutivo, sostiene la hipótesis de procesos a nivel local; y por último, (iii) el modelo integracionista, postula que ambos procesos jugaron un papel destacado en la variabilidad de la población americana (Rodríguez-Cuenca).

Si bien los orígenes y evolución de la población de Sudamérica deben ser conocidos en un estudio de afinidad de rasgos morfológicos con grupos poblacionales, es de vital importancia valorar los movimientos migratorios más recientes que puedan favorecen la miscegenación en poblaciones actuales. Sudamérica ha recibido contingentes poblacionales siguiendo tres patrones bien definidos: el inmigratorio extracontinental, de procedencia europea y de Medio Oriente, ocurrida entre la segunda mitad del siglo XIX y la primera mitad del siglo XX; el intrarregional (tanto inmigratorio como emigratorio), constante debido la proximidad geográfica; y el emigratorio extrarregional, con las salidas hacia Estados Unidos y Canadá (Martínez Pizarro, 2008). Esta llegada de la población europea se aprecia en la tendencia al cambio obtenida en los resultados de los trabajos revisados. Fonseca et al. destacan resultados centrados en poblaciones históricas sugerentes de una progresiva raíz Mongoloide con origen Sinodonte. En la presente revisión, en cambio, las referencias sugieren un origen Mongoloide con influencia Caucasoide (Díaz et al.; Kieser \& Preston), complejo Caucasoide con influencia Mongoloide (García et al.; Goyes et al.; Marcovich et al.; Moreno et al.; Sassi et al.; Villanueva et al.), y de ambos (Aguirre et al. 2006; 2007a; 2007b). La corriente migratoria procedente del continente Africano también se hace presente en los trabajos de Aragón et al. Delgado-Burbano $(2007,2008)$ y Tinoco et al. Todo ello denota la complejidad que implica el estudio de la filogenia humana en Sudamérica como consecuencia de las corrientes migratorias que en los últimos siglos han dado lugar a poblaciones mestizas. Un activo potencial en el estudio reside en la información de cada estructura poblacional y su grado de aislamiento con el fin de conocer el nivel de heterogeneidad de los grupos en relación a su diversidad ancestral. En nuestra investigación, sólo Delgado-Burbano (2007, 2008), Mayhall \& Karp (1981) o Villanueva et al. exponen este tipo de información.

El análisis de los restos óseos para la identificación humana implica el uso de métodos métricos y morfológicos para la reconstrucción del perfil biológico, entre los cuales, la edad, el sexo, la estatura y la ascendencia constituyen el foco principal complementados con el estado de salud, la data de muerte o cualquier otra información que pueda conducir a la identificación (Tinoco et al.). El ancestro com- prende un aspecto esencial; no obstante, resulta más difícil de conocer que el resto, especialmente en poblaciones mixtas. En los casos en que el cráneo se encuentra bien conservado, un análisis de sus rasgos métricos representa el método más empleado para el diagnóstico en contextos forenses; no obstante, cuando el cráneo se encuentra demasiado dañado, la morfología dental es el método preferido para establecer ancestría (Scott \& Turner). Pocas variantes humanas son realmente específicas de una población, con una frecuencia del $100 \%$ en un grupo y de $0 \%$ en otros; sin embargo, el uso de rasgos morfológicos en el contexto forense deben ser lo más específico posible (Edgar, 2009). A pesar de que el premolar Uto-azteca es el único rasgo dental identificable exclusivamente en sólo una población (nativos americanos) (Scott \& Turner), y de que los rasgos morfológicos presentan limitaciones evidentes si son sugeridos en un contexto forense, varios autores han propuesto su empleo en los últimos años (Alsoleihat; Edgar, 2005, 2009, 2013, Edgar \& Ousley; Tinoco et al.). Edgar (2005), plantea que el primer obstáculo a salvar es la asociación irrestricta de rasgos con poblaciones determinadas (Forma de Pala con asiáticas; Carabelli con europeas; etc.); la autora menciona que debe irse más allá de una mera descripción buscando elaborar metodologías estadísticas que permitan someter a prueba la estimación del ancestro. Los trabajos que se basan en una clasificación y comparación de la frecuencia de los rasgos ponderados por el observador tienen una importancia y fiabilidad relativa. El empleo de la correlación, en cambio, no siempre es considerado y tiene un gran poder de deducción (Edgar \& Ousley). La misma Edgar (2013) afirma que la morfología dental, aplicada de la manera clásica, carece de una base estadística fiable en un contexto forense, incumpliendo los reconocidos estándares Daubert (vigentes en la legislación norteamericana e importante referente global), esenciales para admitir este procedimiento como prueba pericial (Edgar, 2013). En esta línea se encuentran trabajos como el llevado a cabo por Edgar (2005) que diferencia Americanos Africanos y Americanos Europeos mediante regresiones logísticas con un $90 \%$ de fiabilidad.

Diecinueve de los 36 trabajos (53\%) hacen mención específica al potencial uso de los rasgos morfológicos dentales como herramientas de identificación forense, todos identificados en la segunda fase cronológica (20012016), 2 de ellos publicados en revistas especializadas en ciencias forenses (Aguayo-Galeano et al.; Tinoco et al.). Entre esos 19 artículos se encuentran los 15 publicados por el Dr. Freddy Moreno (79\%) a través de sus diferentes grupos de investigación. Los mismos autores explican que en Colombia, la investigación en antropología dental se encuentra orientada primariamente no sólo hacia un potencial valor clínico sino también hacia uno forense, por lo que espe- 
ran que estos hallazgos contribuyan a la discusión de su utilidad en estos contextos (Aguirre et al. 2006, 2007a). Los cambios sociopolíticos desarrollados luego del panorama ofrecido por la lucha contra el narcotráfico, el terrorismo, las desapariciones forzadas, el secuestro y el desplazamiento de la población y sus consecuencias (más de 6500 desaparecidos, promedios anuales de 25000 homicidios, inhumaciones ilegales, tortura, 2000 secuestros y cerca de $8000 \mathrm{NN}$ ), favorecieron la inserción efectiva de la antropología forense con inusuales apoyos institucionales del estado y la creación de equipos interdisciplinarios con asignaciones de tareas acordes a las diferentes competencias (Casallas \& Padilla-Piedrahita, 2004). No es dato menor que esta segunda fase cronológica posea las únicas participaciones de antropólogos, todos ellos colombianos dedicados casi de manera exclusiva a la antropología dental (J. V. Rodríguez-Cuenca, C. D. Rodríguez-Flórez y M. E. Delgado-Burbano). Otros países interesados en relacionar rasgos dentales no métricos con identificación forense son Brasil con 2 artículos (Tinoco et al. y Sassi et al. este último en colaboración con Uruguay) y Paraguay (Aguayo-Galeano et al.). Sin embargo, consideramos que, de buscar asignar valor forense a este tipo de rasgos, el muestreo poblacional no puede encontrarse limitado a cotos específicos rurales (etnias), y compartimos las inquietudes de Tinoco et al. cuando refieren que las frecuencias poblacionales usualmente empleadas en antropología y odontología forense deberían ser actualizadas empleando muestras de sujetos vivos más que de colecciones de museos.

Sobre este último punto, resulta curioso el fenómeno observado en Argentina y Perú. Mientras que existe un manifiesto interés por la investigación de la morfología dental de poblaciones históricas en esos dos países, tanto en cantidad de poblaciones estudiadas como en los tamaños muestrales (Fonseca et al.), nuestra revisión acusó sólo dos artículos en poblaciones actuales argentinas -uno focalizado en la Forma de Pala (Devoto et al.) y otro reportando un caso de octava cúspide en un segundo molar temporario (Rodríguez-Flórez et al.)-, y sólo uno en una población actual peruana -53 individuos del norte amazónico (Goaz \& Miller)-. Esto pone en evidencia el manifiesto "poco interés" por actualizar la información con muestreos de este tipo de datos en sujetos vivos de esos países, consideración elemental para poder asignar un valor forense a los resultados. Más allá de toda especulación, Argentina y Perú cuentan con dos de los grupos latinoamericanos más reconocidos en el área (el Equipo Argentino de Antropología Forense EAAF- y el Equipo Peruano de Antropología Forense EPAF-, ambos legado del prominente antropólogo forense estadounidense Dr. Clyde Snow), grupos con prestigiosas contribuciones en la identificación de víctimas de inhumación irregular (Casallas \& Padilla-Piedrahita). Producto de esta experiencia internacional es el denominado "Protocolo modelo para la investigación de muertes sospechosas de haberse producido por violación de los derechos humanos", cuya página 62, en el acápite dedicado a la "Estimación de características raciales" en la observaciones sobre los dientes, menciona a los "Incisivos centrales en forma de pala" como el único indicador no métrico dental que "no requiere equipo específico" y cuya observación "puede ser realizada rápidamente" (Fondebrider \& de Mendonça, 2001). Sin embargo, Edgar \& Ousley aseveran que no ha sido realizada a la fecha ningún tipo de comprobación estadística que permita validar este tipo de estudios como predictores del grupo al que se pretende incluir un determinado individuo, ya que la mayoría se limita sólo a una exposición de la frecuencias de expresión de cada rasgo y a una prueba de distribución. Parra \& Palma (2005) refieren que aunque el EPAF continúa el paradigma de Snow y la misma perspectiva del EAAF, Perú no ha logrado desarrollarse en el ámbito académico, lo que a nuestro entender explicaría la casi absoluta ausencia de autores peruanos en ambas revisiones, más allá del superlativo interés de Estados Unidos y Japón en sus poblaciones históricas (Fonseca et al.). Por su parte, Levin (2015) apunta que en la región es imposible caracterizar el éxito científico de la antropología forense ya que la publicación (el parámetro más relevante para medirlo) ha sido reemplazada por lo "socialmente relevante" de la disciplina. El autor explica esta singularidad en la imposibilidad de "enmarcar estas tareas en el modelo lineal y clásico de una actividad científica que debe validarse como estándar probatorio en juicio". El EAAF no publica sus investigaciones sino en informes propios (Levin); Fonseca et al. explican que la literatura no difundida por los canales tradicionales de publicación comercial o en revistas no incluidas en bases de datos (gris, semi-publicada, invisible, menor o informal), plantea problemas de acceso. Según nuestro entender, el "modelo lineal y clásico" tal y como lo denomina Levin, define hoy no sólo un estándar probatorio sino la posibilidad de que el procedimiento sea reproducible, falsable y verificable, criterios mínimos y básicos para ser considerado científico.

La controversia sobre el empleo de los rasgos morfológicos dentales sigue vigente, situación no sólo observada en Sudamérica sino incluso a nivel global; se conocen sus limitaciones pero aún no ha sido validada con rigor científico su predicción en la estimación del grupo poblacional. Es necesario seguir trabajando en la línea propuesta por Edgar $(2005,2009,2013)$ en sus diversas publicaciones, al amparo de modelos de predicción estadística que permiten poner a prueba si la morfología dental tiene validez como método de estimación de ancestría en un contexto forense o si, al menos, puede emplearse como complementario a otros. 
LÓPEZ-LÁZARO, S.; SOTO-ÁlVAREZ, C.; ARAMBURÚ, G.; RODRÍGUEZ, I.; CANTÍN, M. \& FONSECA, G. M. Research on non-metric dental traits in present south-american populations: State of art and forensic contextualization. Int. J. Morphol., 34(2):580$592,2016$.

SUMMARY: In recent human populations there is a pattern of geographic variation associated to the tooth morphology. In the last decades there has been a remarkable scientific production around the study of non-metric dental traits in order to define the geographic patterns of groups, their history, origin or structure, among others. Between the proposed scopes is the possibility of using the frequency of different traits of expression in a forensic context. We present a review of the literature in MEDLINE, SciELO, and LILACS and REDALYC databases. Full articles in Spanish, Portuguese and English conducting analyzes of dental morphological traits in present South American populations (XX and XXI centuries) were included. Two researchers evaluated the selected articles independently. The search strategies allowed identified 36 studies with a significant participation of Colombia (both authors and samples), followed by Chile and Brazil. While these results demonstrate a robust activity in the field of dental anthropology studying South-American present populations, a reevaluation of the methodologies is suggested if it is to assign a forensic value to dental morphological traits.

KEY WORDS: Dental anthropology; Non-metric traits; South-America; Forensic dentistry.

\section{REFERENCIAS BIBLIOGRÁFICAS}

Aguayo-Galeano, M. A.; Calderón-Giménez, V. M. \& AmarillaGuirland, A. M. Rasgos Morfológicos Dentales de Individuos Paraguayos que acudieron a la Facultad de Odontología de la Universidad Nacional de Asunción, Paraguay, entre los años 2006 a 2011. FOPJ, 3(7):23-31, 2012.

Aguirre, L.; Castillo, D.; Solarte, D. \& Moreno, F. Frequency and variability of five non-metric dental crown traits in the primary and permanent dentitions of a racially mixed population from Cali, Colombia. Dental Anthropol., 19(2):39-47, 2006.

Aguirre, L.; Castillo, D.; Solarte, D.; Moyano, M. \& Moreno, F. Analysis of three non-metric dental traits in a living population from Colombia. Int. J. Dental Anthropol., 10(1):24-35, 2007 a.

Aguirre, L.; D. Castillo, D.; Solarte, D.; Moyano, M. \& Moreno, F. Morfología dental en dentición mixta: Correlación de tres rasgos morfológicos en dientes temporales y permanentes de escolares de Cali, Colombia. Rev. Estomatol., 15(2):10-8, $2007 \mathrm{~b}$

Alsoleihat, F. A New Quantitative Method for Predicting Forensic Racial Identity Based on Dental Morphological Trait Analysis. Int. J. Morphol., 31(2):418-24, 2013.

Alvira, A. M.; Aragón J. F.; Castro J. C.; Rueda L. A. \& Delgado J. E. Características morfológicas de dientes permanentes en dos comunidades colombianas. Univ. Odontol., 21(44):14-21, 2001.

Aragón, N.; Bastidas, C.; Bedón, L.; Duque, P.; Sánchez, M.; Rivera, S.; Triana, F.; Bedoya, N \& Moreno, F. Rasgos morfológicos dentales coronales en dentición temporal y permanente: Distancia biológica entre tres grupos indígenas del Amazonas Colombiano. Rev. Odontol. Mex., 12(1):13-28, 2008.

Blanco, R. \& Chakraborty, R. The genetics of shovel shape in maxillary central incisors in man. Am. J. Phys. Anthrop., 44(2):233-6, 1976.
Bravo, G. E.; Buitrago, Y. A. \& Zarante, I. M. Análisis morfológico dental de dos poblaciones afrocolombianas comparadas con otras poblaciones del mundo. Univ. Odontol., 23(52):21-32, 2003.

Brewer-Carias, C. A.; le Blanc, S. \& Neel, J. V. Genetic structure of a tribal population, the Yanomama Indians. XIII. Dental microdifferentiation. Am. J. Phys. Anthrop., 44(1):5-14, 1976.

Casallas, D. A. \& Padilla-Piedrahita, J. Antropología forense en el conflicto armado en el contexto latinoamericano. Estudio comparativo Argentina, Guatemala, Perú y Colombia. Maguaré, 18:293-310, 2004.

Delgado-Burbano, M. E. Population affinities of African Colombians to Sub-Saharan Africans based on dental morphology. HOMO, 58(4):329-56, 2007.

Delgado-Burbano, M. E. Deciduous Dental Morphological Diversity in Contemporary Colombian Ethnic Groups. Dental Anthropol., 21(2-3):33-45, 2008.

Devoto, F. C., Arias, N. H., Ringuelet, S. \& Palma, N. H. Shovelshaped incisors in a Northwestern Argentine population. $J$. Dent. Res., 47(5):820-3, 1968.

Díaz, E.; García, L.; Hernández, M.; Palacio, L.; Ruiz, D.; Velandia, N.; Villavicencio, J. \& Moreno, F. Frecuencia y variabilidad de la morfología dental en dentición temporal y permanente de un grupo de indígenas Nasa del municipio de Morales, departamento del Cauca. Colomb. Med., 45(1):15-24, 2014.

Edgar, H. J. Prediction of race using characteristics of dental morphology. J. Forensic Sci., 50(2):269-73, 2005.

Edgar, H. J. Testing the Utility of Dental Morphological Traits Commonly Used in the Forensic Identification of Ancestry. En: Koppe, T.; Meyer, G. \& Alt KW (Eds.). Comparative Dental Morphology. Front Oral Biol. Basel, Karger, 2009. pp 49-54. 
Edgar, H. J. Estimation of Ancestry Using Dental Morphological Characteristics. J. Forensic Sci., 58(1):S3-S8, 2013.

Edgar, H. J. \& Ousley, S. D. New approaches to the use of dental morphology in forensic contexts. En: Scott, G. R. \& Irish, J. D. (Eds.). Anthropological Perspectives on Tooth Morphology. Genetics, Evolution, Variation. Nueva York, Cambridge University Press, 2010. pp 510-34.

Ferreira, M. A.; Hespanhol, L. C.; Capote, T. S. O.; Gonçalves, M. A. \& Campos, J. A. D. B. Presencia y morfología del tubérculo molar de acuerdo con la dentición, hemiarco y sexo. Int. J. Morphol., 28(1):121-5, 2010.

Fondebrider, L. \& de Mendonça, M. C. Protocolo modelo para la investigación forense de muertes sospechosas de haberse producido por violación de los Derechos Humanos. Oficina del Alto Comisionado para los Derechos Humanos de las Naciones Unidas. Proyecto MEX/00/AH/10. Primera Fase del Programa de Cooperación Técnica para México. México, 2001. Disponible en http://www.pgjdf.gob.mx/temas/4-6-1/fuentes/11-A-8.pdf

Fonseca, G. M.; Aramburú, G.; Rodríguez, I.; Bollini, G. A.; Atencio, J. P.; Berta, M. J.; López-Lázaro, S.; Cantín, M. \& Lissera, R. G. Development of Research on Morphological Variation of Historical South American Populations Based on Non-metric Dental Traits. Int. J. Morphol., 34(1):11626, 2016.

García, A.; Gustín, F.; Quiñonez, C.; Sacanamboy, L.; Torres, M. H.; Triana, L.; Valencia, D; Rojas, E.; Gómez, J. \& Moreno, F. Caracterización morfológica de incisivos y molares de un grupo de afrodescendientes de Cali, Valle del Cauca (Colombia). Rev. Estomatol., 23(2):17-29, 2015.

Girón, G.; Gómez, P.; Morales, L.; León, M. \& Moreno, F. Rasgos morfológicos y métricos dentales coronales de premolares superiores e inferiores en escolares de tres instituciones educativas de Cali, Colombia. Int. J. Morphol., 27(3):913-25, 2009.

Goaz, P. W. \& Miller M. C. 3rd. A Preliminary Description of the Dental Morphology of the Peruvian Indian. J. Dent. Res., 45(1):106-19, 1966.

Goyes, J.; Guerrero, L.; Narváez, N. \& Moreno, F. Rasgos morfológicos dentales coronarios de caninos temporales y permanentes en un grupo de mestizos de Cali, Colombia. Rev. Colomb. Inv. Odontol., 2(5):1-13, 2011.

Hernández, J.; Villavicencio, J.; Arce, E. \& Moreno, F. Talón Cuspídeo: Reporte de Cinco Casos. Rev. Fac. Odontol. Univ. Antioq., 21(2):208-17, 2010.

Kieser, J. A. \& Preston C. B. The Dentition of the Lengua Indians of Paraguay. Am. J. Phys. Anthrop., 55(4):485-90, 1981.
Kovacevic, A. \& Gruengold, L. Are there any similarities and/ or differences in sex determination methods used in forensic dentistry and paleodontology? Bull. Int. Assoc. Paleodont., 4(1):33-5, 2010.

Levin, L. G. Cuando la periferia se vuelve centro. La antropología forense en la Argentina: un caso de producción de conocimiento científico socialmente relevante. Cuad. Antropol. Soc., 42(2):35-54, 2015.

Marcovich, I.; Prado, E.; Díaz, P.; Ortiz, Y.; Martínez, C. \& Moreno, F. Análisis de la morfología dental en escolares afrocolombianos de Villa Rica, Cauca, Colombia. Rev. Fac. Odontol. Univ. Antioq., 24(1):37-61, 2012.

Martínez Pizarro, J. América Latina y el Caribe: migración internacional, derechos humanos y desarrollo. Comisión Económica para América Latina y el Caribe (CEPAL), Santiago de Chile, Ed. Naciones Unidas, 2008.

Mayhall, J. T. \& Karp, S. A. Size and Morphology of the Permanent Dentition of the Waorani Indians of Ecuador. Can. Rev. Phys. Anthropol., 3(1):55-67, 1981.

Moreno, F.; Moreno, S. M.; Díaz, C. A.; Bustos, E. A. \& Rodríguez, J. V. Prevalencia y variabilidad de ocho rasgos morfológicos dentales en jóvenes de tres colegios de Cali, 2002. Colomb. Med., 35(Supl 1):16-23, 2004.

Moreno, S. \& Moreno, F. Importancia clínica de la antropología dental. Rev. Estomatol., 15(2)Supl. 1:42-53, 2007.

Moreno, S. \& Moreno, F. Incisivos laterales superiores en forma de barril. Reporte de un caso. Rev. Estomatol., 18(2):19$22,2010$.

Ocampo, Á.; Sánchez, J. D.; Cajas, C. H. M. \& Moreno, F. Correlación de diez rasgos morfológicos dentales coronales entre molares deciduos y permanentes en tres grupos étnicos colombianos. Rev. Estomatol., 17(2):7-16, 2010.

Parra, R. C. \& Palma, M. Desde el rincón de los muertos y la memoria de sus familiares. Aportes forenses de la Antropología a los Derechos Humanos. AIBR, 6(39):e1-e15, 2005.

Pinto-Cisternas, J. \& Figueroa, H. Genetic structure of a population of Valparaiso. II. Distribution of two dental traits with anthropological importance. Am. J. Phys. Anthropol., 29(3):339-48, 1968.

Rocha, L.; Rivas, H. \& Moreno, F. Frecuencia y variabilidad de la morfología dental en niños afro-colombianos de una institución educativa de Puerto Tejada, Cauca, Colombia. Colomb. Med., 38(3):210-221, 2007.

Rodríguez, C. \& Moreno, F. Paramolar tubercle on second upper left premolar: a case report. Rev. Estomatol., 14(1):30-4, 2006. 
LÓPEZ-LÁZARO, S.; SOTO-ÁLVAREZ, C.; ARAMBURÚ, G.; RODRÍGUEZ, I.; CANTÍN, M. \& FONSECA, G. M. Investigación de rasgos dentales no métricos en poblaciones sudamericanas actuales: estado de situación y contextualización forense. Int. J. Morphol., 34(2):580-592, 2016.

Rodríguez-Cuenca, J. V. Dientes y Diversidad Humana: Avances de la Antropología Dental. Bogotá, Ed. Guadalupe Ltda., 2003. pp.43-76.

Rodríguez-Flórez, C. D. Antropología dental en Colombia. Comienzos, estado actual y perspectivas de investigación. Antropo, 4:17-27, 2003

Rodríguez-Flórez, C. D. La antropología dental y su importancia en el estudio de los grupos humanos. Rev. Fac. Odontol. Univ. Antioq., 16(1-2):52-9, 2005.

Rodríguez-Flórez, C. D. Occurrence of the Uto-Aztecan premolar trait in a contemporary Colombian Amerindian population. HOMO, 63(5):396-403, 2012.

Rodríguez-Flórez, C. D.; Fonseca, G. M. \& Villalba, M. T. Brief communication: Occurrence of an eighth cusp on primary second mandibular molars of a contemporary Argentinean child. Dental Anthropol., 19(3):73-5, 2006.

Rothhammer, F. \& Aspillaga, E. Breve historia de bioantropología chilena. En: Rothhammer F. \& Llop, E. (Eds.). Poblaciones chilenas: cuatro décadas de investigaciones bioantropológicas. Santiago, Ed. Universitaria. 2004. pp 15-8.

Rothhammer, F.; Lasserre, E.; Blanco, R.; Covarrubias, E. \& Dixon, M. Microevolution in human Chilean populations: IV. Shovel shape, mesial-palatal version and other dental traits in Pewenche Indians. Z. Morphol. Anthropol., 60(2):162-9, 1968.

Rothhammer, F.; Benado, M. \& Pereira, G. Variability of two dental traits in Chilean Indian and mixed populations. Hum. Biol., 43(3):309-17, 1971.

Sassi, C.; Picapedra, A.; Lima, L. N. C.; Massa, F.; Gargano, V.; Francesquini Júnior, L. \& Daruge Júnior, E. Contribución de la antropología dental en la determinación de la identidad uruguaya. Actas Odontol., 10(1):29-45, 2013.

Scott, G. R. \& Turner, C. G. The anthropology of modern human teeth: dental morphology and its variation in recent human populations. Nueva York, Cambridge University Press, 2000.

Tinoco, R. L. R.; Lima, L. N. C.; Delwing, F.; Francesquini Jr, L. \& Daruge Jr, E. Dental anthropology of a Brazilian sample: Frequency of nonmetric traits. Forensic Sci. Int., 258:102.e1e5, 2016.

Turner, C. G. II.; Nichol, C. R. \& Scott, G. R. Scoring Procedures for Key Morphological Traits of the Permanent Dentition: the Arizona State University Dental Anthropology System. En: Kelley, M. A. \& Larsen, C. S. (Eds.). Advances in Dental Anthropology. New York, Wiley-Liss, 1991. pp.13-31.

Villanueva, P.; Quevedo, M.; De Barbieri, Z.; Piñeiro, S.; Herrero, C.; Fernández, M. A. \& Palomino, H. Dental morphological markers as a proxy for ethnicity in Robinson Crusoe islanders. Int. J. Morphol., 33(2):538-43, 2015.
Dirección para Correspondencia:

Gabriel M. Fonseca, DDS, Ph.D.

Facultad de Odontología

Universidad de La Frontera

Avenida Francisco Salazar 01145

Temuco

CHILE

Email: gabriel.fonseca@ufrontera.cl

Recibido : 10-04-2016

Aceptado: 28-05-2016 